\title{
Electronic Health Record
}

\author{
Jivka Vinarova', N. Tzacheva1 \\ 1. New Bulgarian University
}

Summary: This paper describes, from a practitioner's point of view, the concepts, methods and tools involved in the design of a practical and potentially low cost distributed information system, with web-based capabilities, for monitoring glaucoma.

Our experience with existing Hospital Information Systems (HISs) found them unsuitable in the very important monitoring process of patients with glaucoma. Actual Electronic Patient Record (EPR) schemes are more to do with management and appointment simple aspects than with clinical and decision-making processes. In a closer relationship to the specific of the affection, we found that demographic patient databases, usually known as Patient Administration Systems (PASs), have not been designed for being shared or concurrently exploited by different programs or even several replicas of the same program.

Many of the early deficiencies in the process of following-up glaucoma patients by dozens of different ophthalmologists in many independent offices from different clinics (with heterogeneous information recording, not very well managed by the existing office capabilities) could only be solved by specifying, designing and implementing a new EPR scheme in a mixed distributed environment, based on a distributed database as a demographic core (or PAS) of patients with glaucoma. A specialized health record management system, with core functionality in monitoring glaucoma, and core data organized as a distributed database system, has been designed in a bottom-up manner to meet the immediate needs. Its pilot implementation was intentionally kept flexible, taking in account developing standards, to accommodate any anticipated future requirements. Among many other benefits, the new EPR allowed medical doctors (ophthalmologists) to view and modify patient information and records in a safe, flexible and efficient manner. Improvements in all the managerial and decisional aspects (regarding costs and time delays) could also be remarked rapidly.

Keywords: Electronic Patient Record (EPR), Occupational Health Services, Medical Information System (MIS), OHS specialists

\section{Introduction}

The aims and purposes that we had to perform for the development of the already working model of EPR for Occupational Health Services are listed below:

- The model should mirror the practice of an OHS physician and his/her information contacts - with the health provided worker and his GP.

- To observe and pay attention to the documental medical economy, to describe all examined medical and auxiliary parameters and their standardized characteristics. Performing this quite new indexes in the electronic version is facilitated, because of the fact that this is a very new practice and still there are no traditions for applying their paper version.

- To enforce the automated document turnover through the ELECTRONIC PATIENT RECORD (EPR), as the first step of MIS integration on the national level. In this way, it would be possible to make all kinds of the health risk analysis at the working place, distinct health providing and control on the employer.

- EPR should be the basis of the routine report analysis of workers health conditions, as well as allow for substitution of mainly onedimensional with multi-dimensional statistical examinations.

- EPR should combine the center-oriented with decentralized method of approach of examine group health conditions of employees, considering the International Classification of Diseases (ICD).

- EPR and the Information System, destined for, should both allow multi-access of the specialized Database for the purposes of national responsible institutions - mainly for the Center of Hygiene, Medical Ecology and Nutrition and National Register of Occupational Morbidity in Sofia, Bulgaria.

- Stage enlargement of the section "Inquiries (Statistics)" should be also considered - as various algorytmic processing of the collected through work time information. The summarized examined characteristics should be based on the relative contingent of illness in all necessary sections - by sex, by age, by profession, by production work and etc, accounting for selected case-effect relationships and correlations. This will optimize management of socially significant diseases in the relevant professional categories.

- Such information approach will help to promote initiatives directed to workplaces with health risk, work expertise and socio-medical behavior. 


\section{Presentation}

This article is devoted to the Private Health-insurance Company, which maintains Occupational Health Services (OHSs) for insured persons in Sofia, Gabrovo and Bourgas. At the present moment, two OHSs begin their work with our EHR in Sofia.

OHS in Bulgaria are organised in coordination with the "Law for healthy and safety work conditions"(1997) and the Decree N17 (1998) of the Ministry of Health. These regulations are applied everywhere, where the right for healthy and safety work conditions is guaranteed. The role of OHS is to help and consult in an expert way the employers when they apply the law; to register the status of work environment; to control all activities for health protection; to adapt both the employee and environment to each other and to control the injurious factors in the work process.

The functional characteristic of OHS consists of three main groups:

1. Examination, prevention and improvement of a workplace with:

- measuring the factros of the workplace in the following subgroups: physical-chemistry factors, toxico-chemical factors and specialized factors, which are followed by evaluation of health risks from their influences and reorganisation of the workplace, considering the results in an adequate way. It is obligate to maintain the whole specialized infromation for these inconstant factors.

2. Tracing health conditions of an employee with:

- medical and health examination at the moment of entrance of a new employee, preventive medical examinations, preparing individual health records, the periodic generalized health analysis of all employees.

3. Education for prevention from work traumas through:

- preparation for the first and urgent medical aid, self-aid and mutual aid, development and application of rules, instructions and norms for healthy work conditions.

At the beginning of the below described Electronic Patient Record, we began with implementation of the listed functions, their archiving and the usage of specialized information. All of the functions in EPR are performed in consideration with the requirements. Patient records have traditionally been developed to support the clinical practices of individual clinical professions. The records in general describe the planned interventions, observations and relevant specialized test results.

EPR is a computer-stored collection of health information about one person where the items are linked.

MIS and EPR are designed in a way to be the first step in the integration at the national level. The compatibility of computer electronic files for processing information, connected with Occupational Health Services (OHSs) is a significant part of the aims, which are pursued by the reorganizing national health care.

By trying to co-ordinate the specified requirements ad conforming with relatively limited information technology use in this specialized area that is searching for its place among the new forms of health care, we are introducing some basic screens of the working EPR : This is the main screen for work, which is structured by activity types : for patients - on the left and for experts, working in Occupational Health Services (OHSs) - on the right.

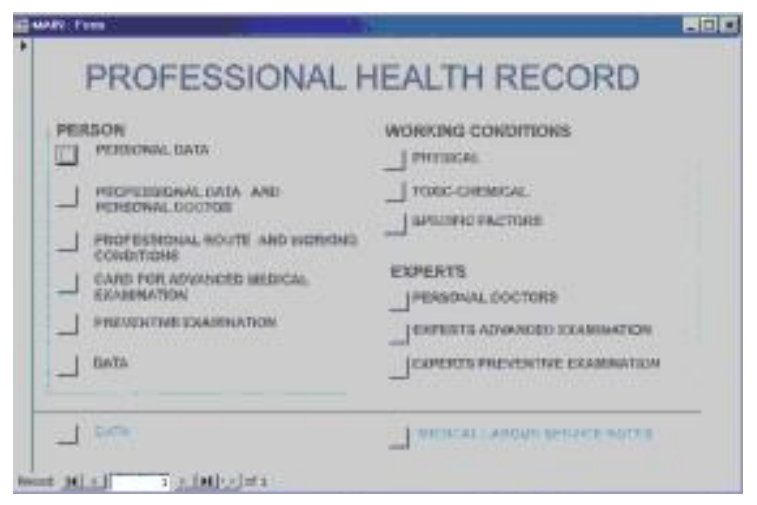

Fig. 1. The main screen. 
Then there is an entry screen for each health insured individual, passing the medical examination. The basic primary information for creation of an electronic record has the following sources :

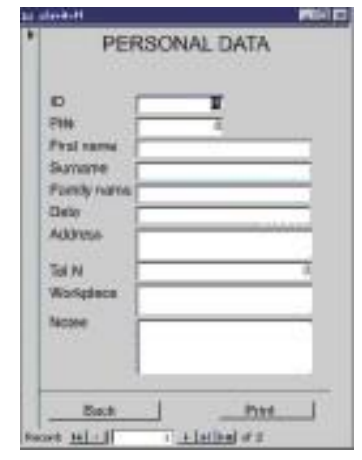

Fig. 2. The entry screen.

1. Passport - professional data - constant administrative-passport data and professional characteristics, through which a reference information in the enterprise's department "Human Resources" and for health-insurance fund is created :

2. Section "Health Condition" - current health and medical information - performed medical examinations by type, decisions of medical commissions and other medical documentation:

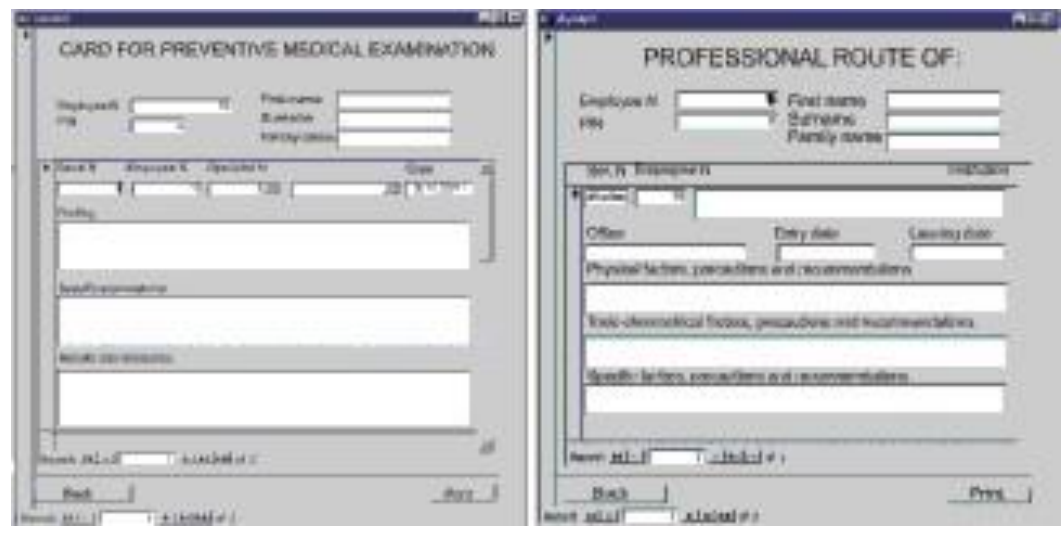

Fig. 3. The "Card for preventive medical examination" screen and Fig. 4. The "Professional Route of:" screen.

In the "Card for preventive medical examination" (Fig.3) there are described the specialized requirements, made by profiles in the field "Finding" from therapeutists, surgeons, neurorlogists, according to the specific characters of the workplace. There are filled in the results - including diagnostic conclusions, which argue the possibility of the employee to work exactly at this workplace.

At the "Proffesional route of:" screen (Fig.4) it is important to describe the factors, which have a constant influence on the work environment, presented in the following groups:

a. Physical factors: electrical safety indicators, noise and vibration measurements, light intensity, temperature, dust. It is obligate to describe the recommended personal and team precautions in the time with a constant or temporary character, including the personal safety measures.

b. Toxico-chemical factors: chemical products in the enviornment, toxical components and their concentration, time of impact. There should be also the record of the recommended and executed precautions in the time with a constant or temporary character.

c. Specific factors: unique features of the work - connected with stress, emotions, work in a conflict environment, including the medical and health professional's recommendations.

3. Information inquire and health indicators:

The "Preventive medical examination" screen (Fig.5) is for registration of regular medical examinations of all employees and for individual visits in a case of a special occasion. At the "Health disease with temporary work disability" screen (Fig.6) there are registered the patient charts for home treatment, days at home and the IDC number. 

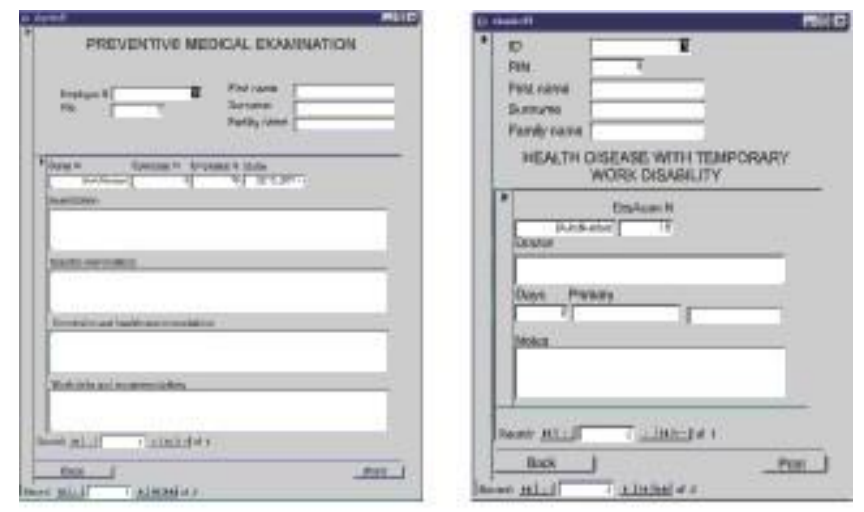

Fig. 5. The "Preventive Medical Examination" screen and Fig. 6. The "Health Disease with Temporary Work Disability" screen.

EPR gives a possibility for information treatment not only from and for the patients, but also for experts to work and apply this information for improvement of health care.

In the "Note" field of Fig.7 every medical specialist can mark her/his observations and work in their uniqeness.

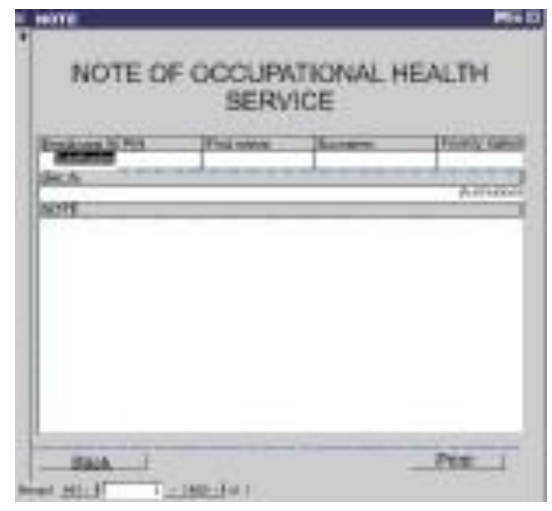

Fig. 7. The "Note" screen.

4. Work of OHS specialists and their connection with GP:

The purpose of the following three screens - Fig.8, Fig.9 and Fig.10 is to create enviornment for information exchange between three types of medical experts, who examine the employee: a doctor at his workplace (with speciality - Occupational medicine), a specialized physician (surgeon) and her/his GP.

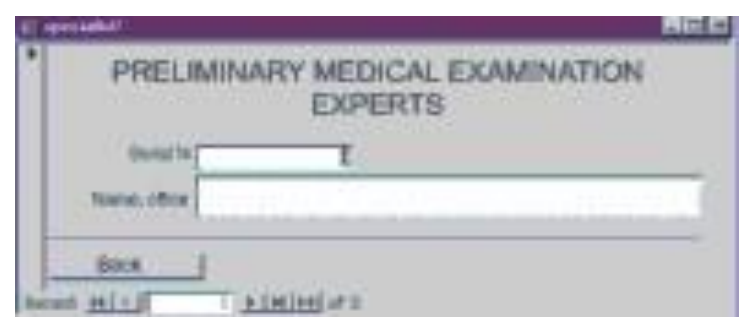

Fig. 8. The "Preliminary Medical Examination Experts" screen.

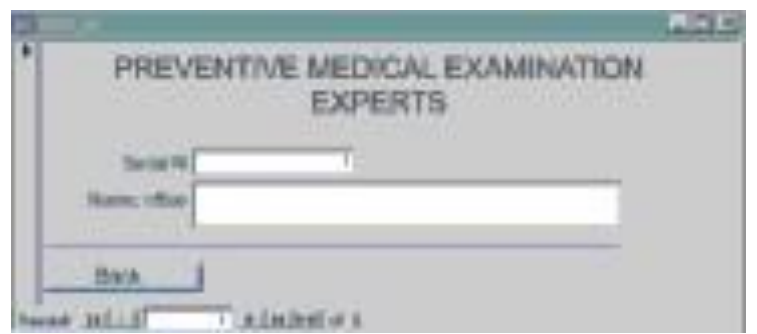

Fig. 9. The "Preventive Medical Examination Experts" screen. 


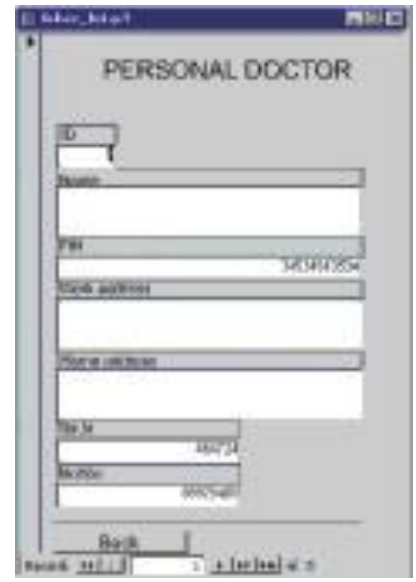

Fig. 10. The "Personal Doctor" screen.

Every entry is connected with the system date, which allows future references and with the administrative-passport data of employed his/her national identifier. Besides, MIS performs entrance "Auto-number" function, which gives the employed his/her system identifier. The searching function could be made by one of these three modes. The "Card for preliminary medical check up", that is performed once before each employment at a new job, is unique for each patient. It is provided with standardized attributes according to the chosen graphical design of the fields. The number of prophylactic check ups are unlimited - after complaining or after OHS's initiative.

\section{Conclusion}

From the big importance for the realization of this project the focus was on the pragmatic aspects of specialized medical and health information and its management use. This information is a very expensive product, which serves for financial reports and analyses at the Health Insurance Fund. It is used by many users in a dialog regime. In future - there are intentions about a packet, epidemiological processing and terminals.

This ELECTRONIC PATIENT RECORD is a stage model of Occupational Health Services functions. It is in a process of optimization the best vision of its formalized structure is still not found, because there is not any paper version to compare with.

The graphical design of EPR is realized on the basis of the present, modern medical electronic documents and it would develop in the practice.

The practical implementation of this product since the end of year 2003, could be generalized in the following frame: in Sofia : CiBank (900 employees) and Bank Biochim (2000 employees), in Mezdra - "Sunytex enterprise" (600 employees), in Gabrovo - "Podem enterprise" (600 employees) and in Bourgas - "Hemus enterprise" (350 employees). The general profit for one year is about 7000 EUR for 4450 employees.

This product is used and applied for education of New Bulgarian University students, the Department "Biomedical science" and there are two new diploma theses, based on it, in the program "Health management and politics".

\section{References, Lectures and Publications}

[1] Vinarova J., Jeliazkova A.: A Variant of Professional Health Record for Computer Processing. Asklepios magazine, ISNN 1310-0637, 2001, volume XIV, pp. 41-44

[2] Lecture courses CST 381 "Medical Computer System" and CST 470 "Modeling affections and processes", NBU, Bachelor`s degree

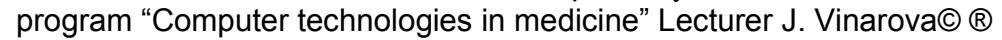

[3] Lecture course MED 128 "Computer systems in the public health sector", NBU, Master's degree program "Healthcare management", Lecturer J. Vinarova@ $\AA$

[4] Collection Cyberculture, NBU edition, author and scientific editing J. Vinarova, ISBN 954-535-227-2, Sofia, 2000 Glossary and manual in Telemedicine - NBU edition, editors of the textbook and translation of the glossary "European Telemedicine

[5] Glossary", Glossary of standards, concepts, technologies and users, edited by DG INFSO, May 2001, editor d-r Luciano Beolchi, J. Vinarova and M. Vukov, ISBN 954-535-269-8, Sofia, 2002

[6] Business documentation of NHIC "Medina"

[7] Regulation № 14/7 August/1998for Occupational services.

[8] Business documentation of "DASIAN", Court order 12977/2003. 